\title{
Advancing Correlative STEM Analysis Methods for FE-SEM
}

\author{
David C. Bell ${ }^{1}$, Natasha Erdman ${ }^{2}$, and Masateru Shibata ${ }^{2}$. \\ 1. Harvard John A. Paulson School of Engineering and Applied Sciences, Harvard University, \\ Cambridge, MA \\ 2. JEOL USA Inc., Peabody, MA
}

Recent developments in SEM column design have led to the ability to produce sub-nm spot sizes even at high probe currents [1], thus pushing the imaging and analytical techniques available in the SEM to approach the resolution and capabilities of a (S)TEM-type instrument. Although the limitations of microanalysis at these spatial resolution requirements stem from the physics of beam-specimen interaction and the volume from which the signal is generated during basic bulk sample observation and microanalysis, use of very thin specimens, similar to TEM, can lead to significant improvements in both imaging microanalysis spatial resolution. This approach has been shown to be successful in for EDS analysis and has been gaining prominence for crystallographic analysis using Transmission Kikuchi Diffraction (TKD) with a traditional EBSD camera [2].

A key advantage of the SEM is the fact that the imaging of ultra-thin specimens is conducted at $30 \mathrm{kV}$ or less, unlike the TEM that is traditionally operated at $80-300 \mathrm{kV}$. The direct advantage of lowering the beam energy is the increased inelastic scattering cross-section that provides substantially improved contrast for imaging. Another advantage for imaging at $30 \mathrm{kV}$ is the ability to substantially reduce specimen damage, especially for materials sensitive to knock-on effects (such as carbon based materials).

In this paper we will discuss various avenues for specimen analysis using ultra-thin specimens in SEM. We will discuss different techniques available to obtain STEM-in-SEM images (from converter holders to dedicated multi-segment detectors); we will also address the relevant sample preparation techniques to produce both superb imaging and microanalysis. The presentation will focus on application of the technique for a variety of specimens - from catalysts and nano-grained metals to biological tissue specimens, viruses, nanoparticles and more (Figure 1). We will also compare the data obtained via STEM-in-SEM to a dedicated S(TEM) instrument operated at low accelerating voltage. We will also present the results and challenges associated with collecting nano-analysis data with ultra-thin specimens. In particular, we'll show that using STEM-in-SEM in combination with EDS allows examination of 2-5 nm sized particles, whereas utilization of the TKD technique with ultra-thin and nanoparticle specimens allows to extend the limit of EBSD analysis down to few 10s of nanometers (Figure 2).

These techniques for nanoscale imaging and analysis in SEM have a potential to greatly enhance the research efforts employing SEM, provided that sufficiently thin specimens can be obtained.

\section{References:}

[1] D.C. Bell and N. Erdman (ed.), 2013. Low Voltage Electron Microscopy - Principles and Applications, Wiley.

[2] N. Erdman et al. 2014. Microscopy and Microanalysis 20 (S3), 864-865 

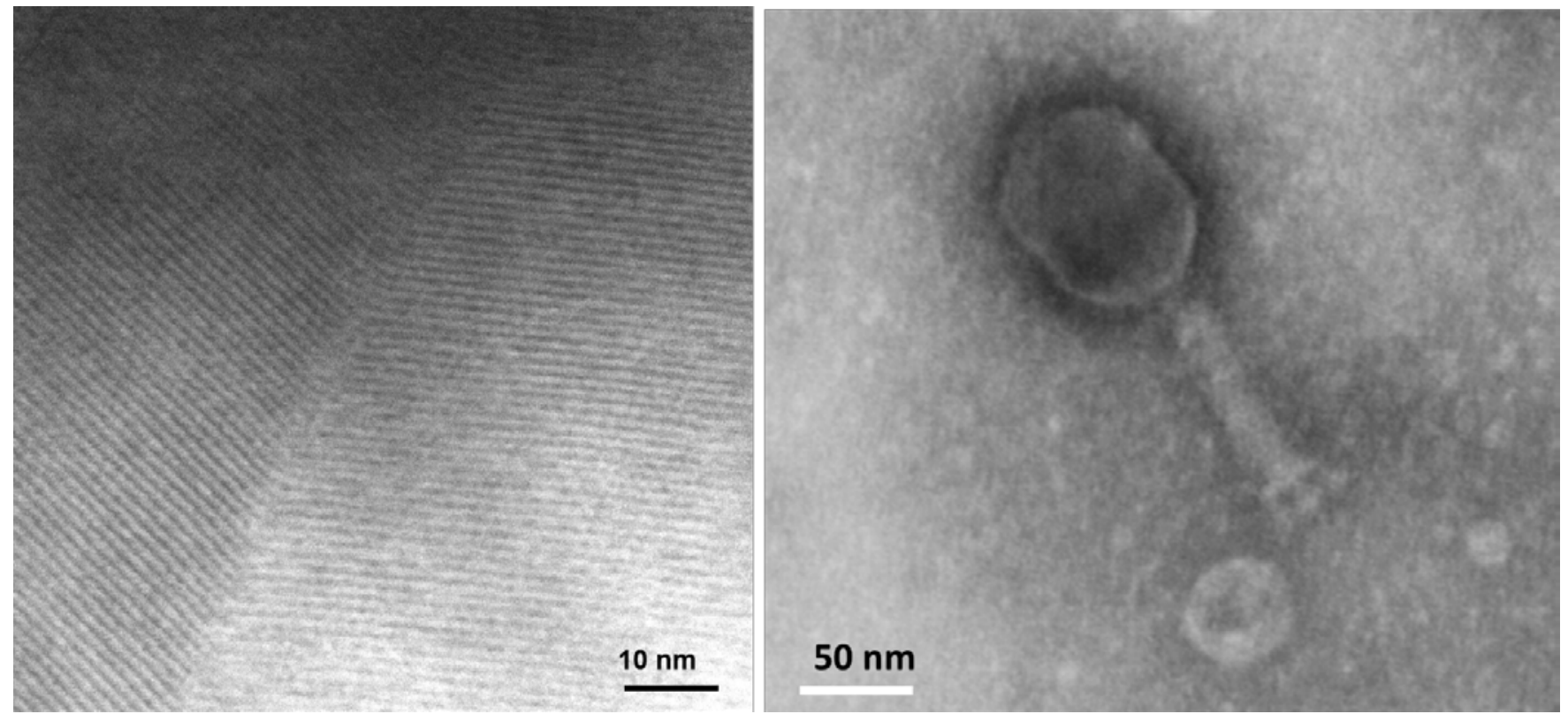

Figure 1. Examples of STEM-in-SEM imaging: left - Faujasite zeolite; right - Enterobacteria Phage T4.

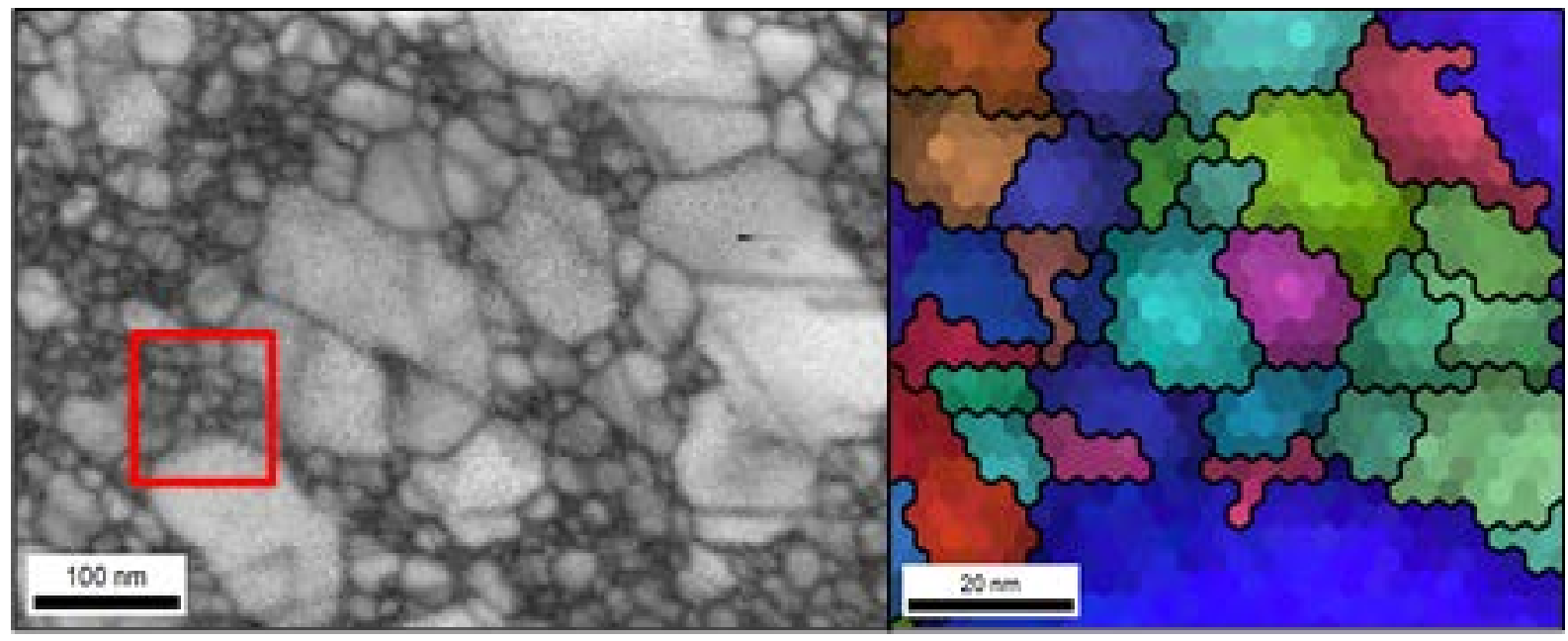

Figure 2. Image quality map and corresponding IPF map (ND) from evaporated Au film analyzed in TKD configuration. Red square shows the analysis area on the right. 